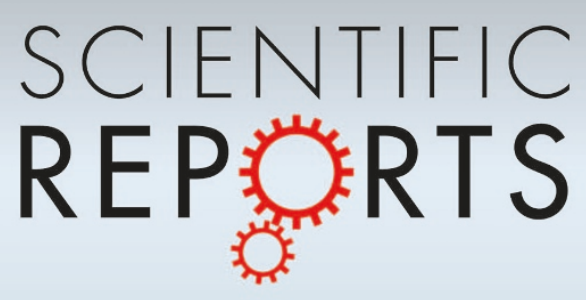

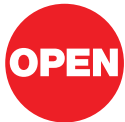

SUBJECT AREAS:

CHANNELS

GROWTH AND DEVELOPMENT BIOPHYSICS

ZEBRAFISH

Received

13 March 2012

Accepted

11 June 2012

Published

26 June 2012

Correspondence and requests for materials should be addressed to M.W. (watanabe-m@ fbs.osaka-u.ac.jp)

\section{Polyamine sensitivity of gap junctions is required for skin pattern formation in zebrafish}

\author{
Masakatsu Watanabe, Daisuke Watanabe \& Shigeru Kondo
}

Graduate School of Frontier Biosciences, Osaka University 1-3 Yamadaoka, Suita, Osaka 565-0871, Japan.

Gap junctions allow the direct and bidirectional transfer of small molecules between cells. Polyamine sensitivity, which has been observed for a certain gap junction in vitro, confers rectification property to gap junction. Here we report that the polyamine sensitivity of gap junctions in vivo is crucial for skin pattern formation in zebrafish. Transgenic experiments have revealed that several connexin genes were able to rescue the spot phenotype of mutant zebrafish. Mutational analyses of the $\mathrm{N}$-terminal region of connexins revealed that the ExxxE motif, a hypothetical polyamine-binding site, was important for connexin's role in pattern formation. Ectopic expression of spermidine/spermine $N^{1}$-acetyltransferase (SSAT), a polyamine metabolic enzyme, also caused stripe pattern changes, which further indicates that the polyamine sensitivity of gap junctions is crucial. This is the first report to show that polyamine sensitivity has a physiologically relevant function and is related to skin pattern formation in animals.

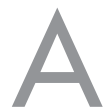

gap junction is an intercellular channel that forms between two neighbouring cells to allow small molecules $(<1,000 \mathrm{Da})$ such as ions, small metabolites and second messengers to translocate from cell to cell $^{1-3}$. Each gap junction is composed of two connexons, which are made up of six connexin molecules. In mammals, approximately 20 connexin genes exist in the genome, whereas approximately 40 connexin genes are predicted to be present in the teleost genome. Connexin has intracellular N- and C-terminal domains, four transmembrane domains, two extracellular domains and one cytoplasmic loop domain. The gating of the channel depends on several conditions including transjunctional voltage, intracellular conductance and regulation by polyamine. The N-terminal domain is predicted to have a voltage sensor for the intracellular potential of the cell and to control the open/closed state of the channel ${ }^{4}$. Recent studies clearly revealed that the N-terminal domain of connexin in fact forms a plug structure that controls the open/closed state of the channel ${ }^{5-7}$.

The flow of small molecules through gap junction is usually bidirectional; however, the unidirectional flow of small molecules has also been observed in heterotypic gap junctions ${ }^{8}$. The mechanism underlying unidirectional flow is related to differences in pore sizes and voltage sensors for each connexon molecule. In the case of rat Connexin40 (rat-CX40), unidirectional flow is induced by polyamine, which may bind to the E9 and E13 residues in the N-terminal domain of the connexin molecule ${ }^{9-11}$. Polyamine is a small molecule that functions as an inhibitor of several channels, such as inwardly rectifying potassium channels (Kirs) ${ }^{12,13}$.

The zebrafish, a model organism for both developmental and genetic studies ${ }^{14}$, has a pattern of stripes on its body (Fig. 1b). Each stripe is delineated by two types of pigment cells, melanophores (black pigmented cells) and xanthophores (yellow pigmented cells). The development and differentiation of these pigment cells have been well studied, and several genes, including mitfa, $c$-kit, $c$-fms, and ednrb1, were identified and analysed in this fish ${ }^{15-18}$. The zebrafish is also known as a model for the study of cell-autonomous pattern formation. Melanophores and xanthophores interact with one another throughout their development, and these interactions are important for generating their final pattern ${ }^{19-24}$. Recently, our group revealed that the membrane potential of melanophores is a key factor in the development of clear boundaries between melanophores and xanthophores $^{25,26}$. The molecule that is needed to maintain membrane potential is the inwardly rectifying potassium channel J13 (Kir7.1). Without Kir7.1 function, the regions of melanophores and xanthophores on fish skin are mingled, and their boundary is obscure.

Among the skin pattern mutants of zebrafish, leopard has been considered the most important one for pattern formation studies ${ }^{20,22,27}$ because this fish has a pattern of spots instead of stripes (Fig. 1c). Previously, we identified connexin41.8 (cx41.8) as the gene responsible for the leopard zebrafish ${ }^{28}$. Among the leopard alleles, $c x 41.8^{t 1}$, which exhibits a recessive phenotype, has a nonsense mutation in $c x 41.8$, whereas the dominant alleles $c x 41.8^{\text {tq270 }}$ and 


2 kb
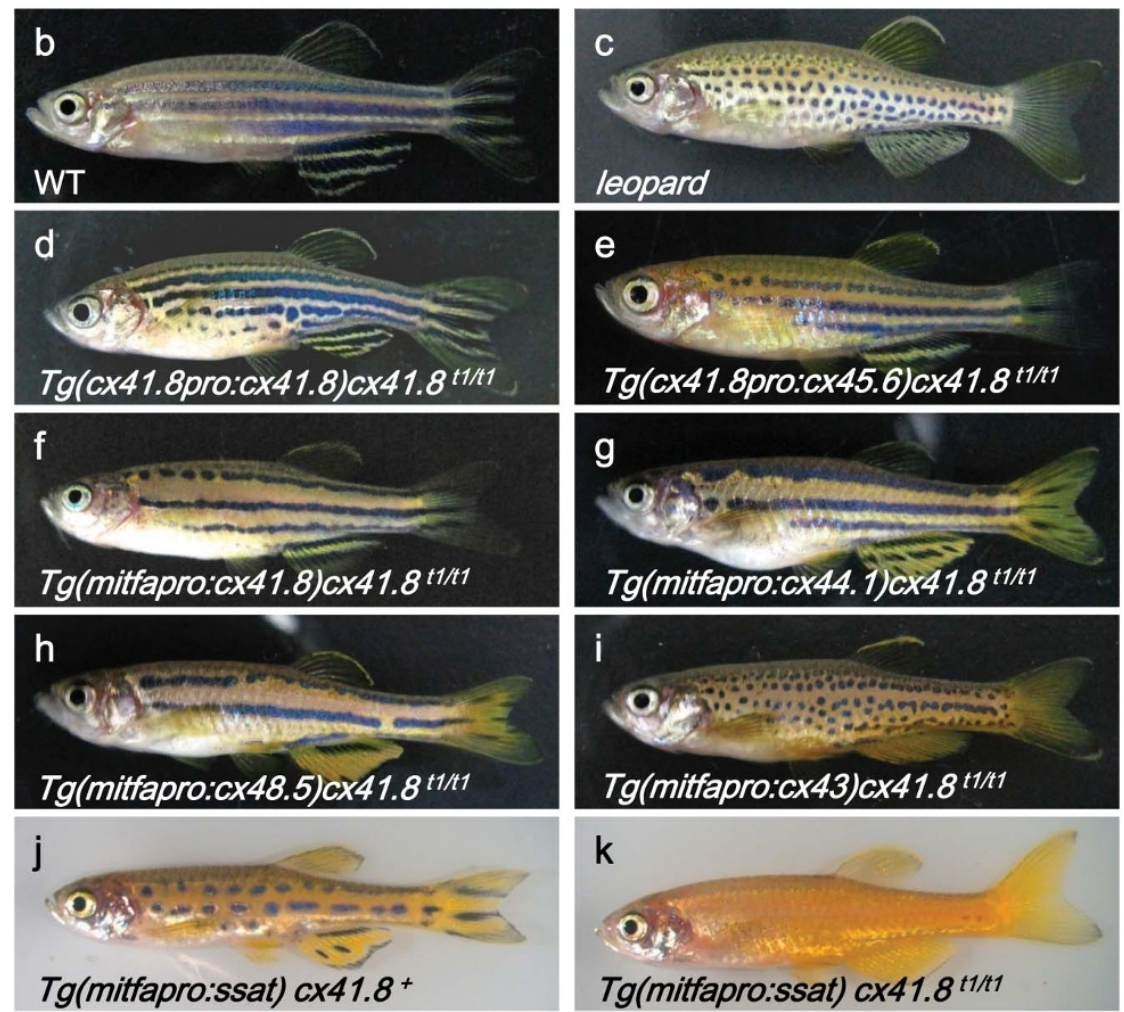

Figure 1 Transgenic zebrafish. (a) Genome structure around $c x 41.8$ (upper line) and plasmid constructs in a pTol2 vector containing cx41.8pro (yellow box) and mitfapro (orange box). Purple boxes, $c x 41.8$ exons; blue box, open reading frame of $c x 41.8$; black box and "TP", Tol2 transposon sequence; red box, target gene. (b) Wild-type (WT) zebrafish. (c) leopard zebrafish. (d-i) Transgenic (Tg) zebrafish with cx41.8pro:cx41.8 (d), cx41.8pro:cx45.6, (e), mitfapro:cx41.8 (f), mitfapro:cx44.1 (g), mitfapro:cx48.5 (h) and mitfapro:cx43 (i). ssat was ectopically expressed in melanophores from wild-type fish $(\mathrm{j})$ and leopard fish $(\mathrm{k})$.

$c x 41.8^{t w 28}$ have missense mutations in the transmembrane regions of cx41.8. $c x 41.8$ is expressed in both melanophores and xanthophores and functions to positively regulate the number of melanophores and negatively regulate the number of xanthophores ${ }^{20,27,29}$.

In this study, we generated a series of transgenic zebrafish to identify the common features of various connexins that are required for zebrafish skin pattern formation. We found that polyamine sensitivity of gap junctions, which might induce rectification properties in gap junctions, is crucial for pattern formation in zebrafish.

\section{Results}

Effect of connexin genes on pattern formation. Using the Tol2transposon system ${ }^{30,31}$, a connexin gene was integrated into leopard zebrafish as described ${ }^{29}$; the connexin gene was expressed under the control of the $c x 41.8$ promoter (cx41.8pro) or the microphthalmiaassociated transcription factor a (mitfa) promoter (mitfapro) (Fig. 1a). cx41.8pro regulates gene expression in both melanophores and xanthophores, whereas mitfapro controls expression only in melanophores of adult fish ${ }^{29}$. We used the $c x 41.8^{t 1}$ allele for the transgenic experiments here because this allele is a functional null allele; thus, endogenous cx41.8 would not influence the function of the transgenes. The phenotypes of the transgenic zebrafish were examined in the resulting F1 generations. $T g$ (cx41.8pro:cx41.8) fish were almost completely rescued by the transgene (Fig. 1d). When the promoter was changed to mitfapro, the resulting pattern was changed slightly with narrower stripes and wider inter-stripe distances (Fig. 1f). The difference in phenotypes between $\operatorname{Tg}(c x 41.8$ pro:cx41.8) and $\mathrm{Tg}$ (mitfapro:cx41.8) fish was most likely caused by differences in gene expression in xanthophores; presumably, expression of $c x 41.8$ in xanthophores decreased the number of xanthophores (see ref. 29 and the Discussion).

We assessed the effect of other connexin genes on pattern formation based on the phenotypic change from spots (Fig. 1c) to stripes (Fig. 1d and f). The promoter sequence used in each transgenic experiment was chosen based on the available restriction enzyme sites in each gene. Based on these experiments, we concluded that $c x 41.8$ (Fig. 1d and f), $c x 45.6$ (Fig. 1e), $c x 44.1$ (Fig. 1g) and $c x 48.5$ (Fig. $1 \mathrm{~h}$ ) rescued the leopard phenotype, whereas $c x 43$ (Fig 1i) failed to do so. We also generated transgenic zebrafish using $c x 27.5, c x 32.2$ and $c \times 33.8$ and found that these genes did not rescue the leopard phenotype (Fig. 2b right). Fig. 2a illustrates the structure of connexin, and the phylogenetic tree in Fig. $2 b$ represents relationships among connexin genes in zebrafish ${ }^{32}$. The connexin genes that were able to rescue the leopard phenotype are clustered (Fig. 2b; Cx48.5-Cx50.5). Here we have referred to this cluster as the "Cx41.8 cluster".

Domain replacement and modification experiments. Because $c x 43$ did not rescue the leopard phenotype in the transgenic experiment, we next examined the differences between $c x 41.8$, which was capable 


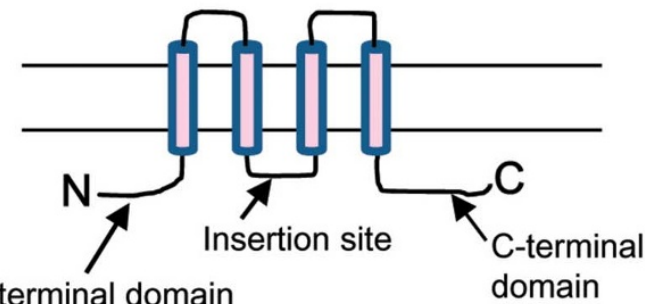

$\mathrm{N}$-terminal domain

C

Cx41.8

(10

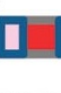

$\mathrm{N} 43 \mathrm{C} \times 41.8$

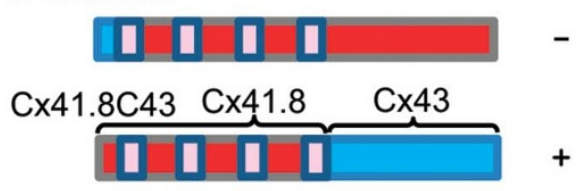

Cx41.8IMM

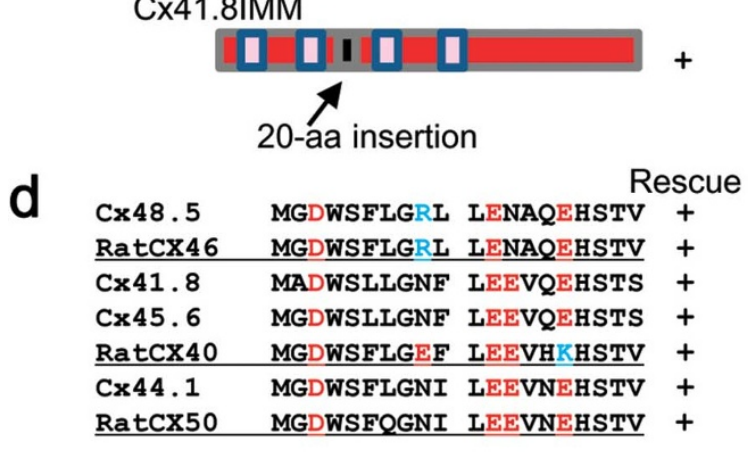

e

\begin{tabular}{lll} 
& & \multicolumn{2}{r}{ Rescue } \\
Cx41.8 & MADWSLLGSF LEEVQEHSTS & + \\
N43Cx41.8 & MGDWSALGRI LDKVQAYSTA - \\
Cx43 & MGDWSALGRL LDKVQAYSTA - \\
Cx43SEEH & MGDWSALGSL LDEVQEHSTA - \\
RatCX40 & MGDWSFLGEF & LEEVHKHSTV \\
RatCX40* & MGDWSFLGKF LEKVHKHSTV & - \\
RatCX43 & MGDWSALGKL LDKVQAYSTA & -
\end{tabular}

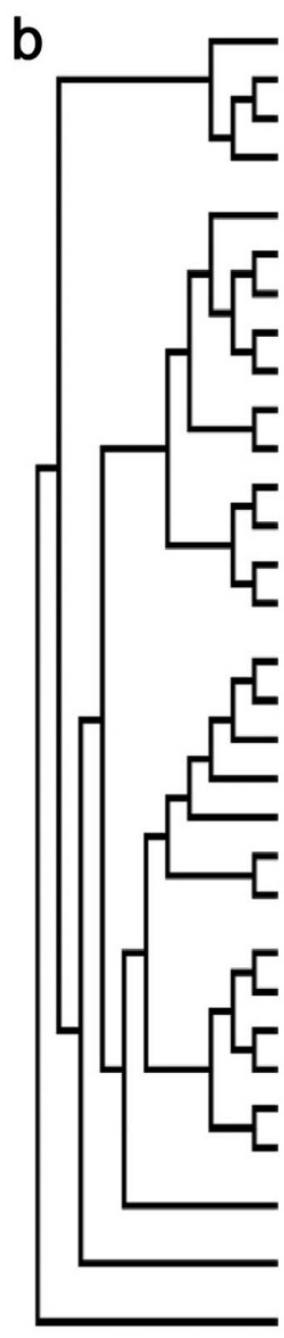

Cx52. 8

Cx44.2

Cx43. 4

Cx47.1

Cx28. 8

Cx35.4

Cx34. 4

Cx28. 6

Cx30.9

Cx33. 8

Cx27.5

Cx52.9

Cx55. 5

Cx52. 6

Cx52. 7

Cx32. 2

Cx32. 3

Cx28. 9

Cx28.1

Cx34. 5

$\mathrm{Cx} 43$

Cx40. 8

Cx48. 5

Cx39. 9

Cx41. 8

Cx45. 6

Cx44.1

Cx50.5

CX39.4 MSRADWGFLEHL LEEGQEYSTG

Cx35

Cx46.8

MS-WSFLTRL LEEIQHHSTS

MS-WSFLTRL LDEISNHSTF

MS-WSFLTRL LDEISNHSTF

MS-WSFLTRL LEEIHNHSTF

MN-WGFLENV LSGVNRYSTV

MD-WKTFQAL LSGVNKYSTA

MN-WAFLQGL LSGVNKYSTA

MN-WSGLQSL LSGVNQYSTV

MN-WLSLEVL LGGVSQYSTV

MS-WGALYAQ LGGVNKHSTS -

MN-WASFYAV ISGVNRHST- -

MGDWNFLGGI LEEVHIHSTM

MGDWNFLGGI LEEVHIHSTM

MGDWNLLGSI LEEVHIHSTI

MGDWNLLGSI LEEVHIHSTI

MGDWGFLSAL LDKVQSHSTV -

MGDWGFLSSL LDKVQSHSTV

MGEWGFLSKL LDKVQSHSTV

MGDWGFLSKL LDKVQSHSTS

MGEWDFLGRL LDRVQTHSTV

MGDWSALGRL LDKVQAYSTA -

MGDWSALGKL LDKVQAYSTA

MGDWSFLGRL LENAQEHSTV + MGDFSSLGKL LESAQEHSTV MADWSLLGNF LEEVQEHSTS + MGDWSLLGNF LEEVQEHSTS + MGDWSFLGNI LEEVNEHSTV + MGDWSFLGRL LENAQEHSTV

MGEWTILERL LEAAVQQHST

MAKQATSEVI FITLNHNITL

Figure $2 \mid$ Summary of rescue experiments. (a) Connexin structure (see also the Introduction). The insertion site in Cx41.8IMM is indicated. (b) Phylogenetic tree of zebrafish connexins and comparison of their N-terminal domains. (c) Schematic diagrams of connexin chimeras and modified connexin. Cx41.8, wild-type zebrafish Cx41.8; N43Cx41.8, N-terminal domain of Cx41.8 was replaced with that of Cx43; Cx41.8C43, C-terminal domain of Cx41.8 was replaced with that of Cx43; Cx41.8IMM, 20 amino acid (aa) residues ( $2 \times$ Myc tag sequences) were inserted into the cytoplasmic loop domain of Cx41.8 (black box). Red and pink boxes illustrate amino acid sequences from Cx41.8. Pink boxes indicate the transmembrane regions. Blue boxes indicate amino acid sequences from $\mathrm{Cx} 43$. (d) N-terminal sequences of rat and zebrafish connexins from the Cx41.8 cluster that were analysed for rescue of the leopard phenotype. (e) A comparison of $\mathrm{N}$-terminal sequences from mutant connexins and wild-type connexins. $\mathrm{N} 43 \mathrm{Cx} 41.8$ is the $\mathrm{Cx} 41.8$ mutant with the $\mathrm{N}$-terminal region of $\mathrm{Cx} 43$; Cx43SEEH is the $\mathrm{Cx} 43$ mutant with 4 aa substitutions; rat-CX40* is the rat-CX40 mutant in which the conserved E9 and E13 residues have been substituted with lysine residues. The results from rescue experiments are shown to the right of each panel (b-e; +, able to rescue the leopard phenotype; -, unable to rescue the leopard phenotype). Rat connexins are underlined (d, e). Red characters indicate acidic residues, and blue characters indicate basic residues ( $b, d$ and $e$ ).

of rescue, and $c x 43$. The $\mathrm{N}$-terminal domain and C-terminal domain in $\mathrm{Cx} 41.8$ were each replaced with those of $\mathrm{Cx} 43$ (Fig. 2c). In parallel, we modified the cytoplasmic loop domain of $\mathrm{Cx} 41.8$ by inserting an extra 20 amino acid residues consisting of a $2 \times$ Myc tag sequence (Cx41.8IMM). We then examined the effect of each construct on pattern formation. We found that Cx41.8C43 and Cx41.8IMM rescued the leopard phenotype, but $\mathrm{N} 43 \mathrm{Cx} 41.8$ did not, indicating that the $\mathrm{N}$-terminal domain of $\mathrm{Cx} 41.8$ is important for skin pattern formation in zebrafish.

The ExxxE motif is the determinant of the Cx41.8 cluster. We then compared the amino acid sequences of the $\mathrm{N}$-terminal domains of the zebrafish connexins (Fig. 2b). We found that the ExxxE motif in this region was highly conserved in the Cx41.8 cluster. In addition, we also examined the rescuing activity of the rat connexins rat-CX40 and rat-CX43, which are orthologs for zebrafish $\mathrm{Cx} 41.8$ and $\mathrm{Cx} 43$, respectively, and found that rat-CX40 also rescued the leopard phenotype, although rat-CX43 failed to do so (Fig. 2d, e). These results indicate that the functional similarities of connexins are well conserved between rat and zebrafish. We note that rat-CX40 has polyamine sensitivity, and the E9 and E13 residues in the Nterminal domain of rat-CX40 are predicted to be the residues that are sensitive to polyamine ${ }^{9,10}$. We thus substituted K9 and K13 for E9 and $\mathrm{E} 13$, respectively, in rat-CX40, which destroys the spermine 
sensitivity of rat-CX40 but barely affects channel function. As expected, our results clearly showed that the rat-CX40E9,13K mutant did not rescue the leopard phenotype (Fig. 2e; ratCX40*), indicating that the spermine sensitivity of this gap junction is important for pattern formation in zebrafish. Furthermore, we made a mutant $\mathrm{Cx} 43, \mathrm{Cx} 43 \mathrm{SEEH}$, which mimics the N-terminal sequence of $\mathrm{Cx} 41.8$ by introducing the amino acid substitutions R9S, K13E, A16E and Y17H into Cx43 (Fig. 2e). We found that these amino acid substitutions were not sufficient to produce Cx41.8-like function in this version of $\mathrm{Cx} 43$ because the Cx43SEEH mutant did not rescue the leopard phenotype (Fig. 2e). This result is consistent with a previous report that rat-CX43 with $\mathrm{K} 9,13 \mathrm{E}$ substitutions does not exhibit rectification property in vitro and that the E9 and E13 pair is not the only determinant of the rectification property of gap junctions ${ }^{10}$.

We also compared the $\mathrm{N}$-terminal sequences of connexins in the $\mathrm{Cx} 41.8$ cluster from various organisms and found that the consensus motif ExxxE was also well conserved (Fig. 2d and see Supplementary Fig. S1 online). This degree of conservation suggests the importance of the ExxxE motif for the function of these connexins. We again used several of these rat connexins for rescue experiments. RatCX46, which is an ortholog to zebrafish Cx48.5, and rat-CX50, which is an ortholog to zebrafish $\mathrm{Cx} 44.1$, rescued the leopard phenotype, as did rat-CX40. These results indicated that the function of members of the Cx41.8 cluster might be well conserved between zebrafish and rat.

SSAT changes the skin pattern in zebrafish. To confirm that a polyamine such as spermidine/spermine was involved in zebrafish pattern formation, we introduced zebrafish-ssat ${ }^{33}$, a metabolic enzyme of spermidine and spermine, into the zebrafish genome. Ectopic expression of ssat in melanophores of fish with a wild-type background generated a spotted pattern (Fig. 1j), whereas scattered melanophores (i.e., the absence of spots or stripes) were observed in leopard background fish (Fig. 1k). These results indicated that polyamine is involved in the skin pattern formation of zebrafish and that the polyamine sensitivity of gap junctions may be crucial for pattern formation in zebrafish.

\section{Discussion}

Here, we have shown that polyamine binding of $\mathrm{Cx} 41.8$ is required for skin pattern formation of zebrafish. Based on the link between polyamine binding and the rectification property of rat-CX40 gap junction ${ }^{9,10}$, we suggest that zebrafish $\mathrm{Cx} 41.8$ may also have a rectification property. To confirm its presence, we tried to perform an electrophysiological assay with $\mathrm{Cx} 41.8$ using mammalian cultured cells. We could not, however, detect gap junctional intercellular communication (GJIC) between transfected cells in culture and thus could not examine the rectification property of $\mathrm{Cx} 41.8$ (data not shown). We also examined the protein localisation of $\mathrm{Cx} 41.8$ in cultured cells, but we could not detect the membrane localisation of a Cx41.8:EGFP fusion protein or even of unmodified $\mathrm{Cx} 41.8$ (data not shown). We believe that the amount of $\mathrm{Cx} 41.8$ that is localised to the cell membrane is too small in mammalian cultured cells and that some unidentified factor(s) might be required for $\mathrm{Cx} 41.8$ localisation to the cell membrane, as was observed for zebrafish $\mathrm{Cx} 40.8^{34}$. Because we could not examine the rectification property of $\mathrm{Cx} 41.8$ directly, we used the ortholog rat-CX40 to suggest that the rectification property of gap junction may be required for skin pattern formation in zebrafish.

At present, it remains unclear what type of function the rectification of gap junctions leads to and when and where this property is required in zebrafish. Indeed, the role of gap junctions in pattern formation in zebrafish has not been fully elucidated ${ }^{29}$. The expression of $c x 41.8$ is detected in melanophores and xanthophores based on RT-PCR analysis ${ }^{28}$; this localisation is also supported by transgenic experiments $^{29}$. The role of $\mathrm{Cx} 41.8$ in both melanophores and xanthophores was predicted based on observations of mutant fish and transgenic fish ${ }^{27-29}$. In fish expression the leopard mutant alleles $c x 41.8^{t 1}$ and $c x 41.8^{t q 270}$, the number of melanophores is smaller than that in wild-type fish, indicating that the loss of function of $\mathrm{Cx} 41.8$ causes a reduction in the number of melanophores. When $\mathrm{Cx} 41.8$ was ectopically expressed in melanophores, the number of melanophores increased, although the area that was covered by the melanophores (i.e., the black stripes) was still smaller in the transgenic fish (Fig. 1f) than that in wild-type fish (Fig. 1b). Cx41.8 expression in xanthophores is likely required to make normal black stripes because the number of xanthophores and the area of xanthophores in $\mathrm{Tg}$ (mitfapro:cx41.8) fish are both larger than those in the wild type ${ }^{29}$. Taken together, Cx41.8 functions to control the number of pigment cells, both melanophores and xanthophores, on the skin surface, and the rectification property of gap junction might be important for this function.

Concerning the existence of polyamines in pigment cells, this small molecule should be localised to and function at the intracellular membrane of melanophores to bind to gap junctions. Our previous work revealed that kir7.1, a member of the inwardly rectifying potassium channel, is responsible for one of the zebrafish skin pattern mutants, jaguar, which shows a broader stripe pattern than that of wild-type fish ${ }^{25}$. For Kir7.1 channels, polyamine is required to induce rectification property by binding to the pore domain of the channel ${ }^{12}$, where polyamine blocks the flow of ions from the inside to the outside of the cell. kir7.1 is found in both melanophores and xanthophores based on RT-PCR ${ }^{25}$, but its expression in melanophores is sufficient for skin pattern formation in zebrafish ${ }^{26}$. These findings support the idea that a polyamine such as spermine could be distributed around the cell membrane of melanophores and that a polyamine may function to induce the rectification properties of both Kir7.1 and gap junction.

The results of transgenic experiments using ssat, which encodes a metabolic enzyme of spermidine/spermine, also supported the existence and functional effects of a polyamine in melanophores. We note that zebrafish carrying the mitfa:ssat transgene in the WT background showed an intermediate phenotype between those of leopard (spots) and jaguar (broad stripes) fish, namely, larger spots with larger inter-spot distances. Furthermore, the phenotype of mitfa:ssat transgenic fish in the leopard background resembled that of leopard/jaguar double-homozygous mutant fish ${ }^{27}$. Although the significance of polyamine sensitivity for gap junction communication relying on the function of $\mathrm{Cx} 41.8 \mathrm{~N}$-terminus remains unclear in vivo, these observations also support the function of spermine in pigment cells.

In mammals, the overlapping expression of connexins occurs in many tissues such as the heart, retina, inner ear, skin and reproductive organs ${ }^{35-41}$, and complementation experiments have been carried out to determine the functional differences between connexin genes $^{42}$. Based on our greater understanding of the relevance of polyamine sensitivity to gap junction function, it would be of interest to carry out a functional comparison among the Cx41.8 cluster members CX40, CX46 and CX50 for further understanding of gap junction function.

Our study provides new insight into gap junction research. In particular, the rectification property of gap junction should be considered when the function of gap junction is examined. Further analysis will reveal the role of the rectification property of gap junction in pattern formation and in other biological phenomena.

\section{Methods}

Fish. Zebrafish (Danio rerio) were bred and maintained under standard laboratory conditions ${ }^{43}$. Images were acquired using a Leica MZ16FA microscope after the fish were anaesthetised with MS222 (Sigma). All experiments were approved by the Animal Experiments Committee of Osaka University, Japan. 
cDNA synthesis and plasmid construction. Total RNA was purified from fish fins and brains using an RNeasy Mini Kit (Qiagen). First-strand cDNA was synthesised with Superscript III (Invitrogen) and oligo d(T) ${ }_{18}$ primer (Hokkaido System Science). The Tol2 plasmid with cx41.8pro and mitfapro was described previously ${ }^{29}$. Each connexin gene was amplified with KOD-Fx DNA polymerase (Toyobo) with specific primer sets in which a restriction enzyme recognition sequence was added at the $5^{\prime}$ end of each primer. Zebrafish ssat (sat1b; XP_696280) was also cloned into the pTol2mitfapro vector using the same strategy.

Transgenic zebrafish. Tol2 plasmid $(30 \mathrm{ng} / \mu \mathrm{l})$ and transposase mRNA (10 $\mathrm{ng} / \mu \mathrm{l})$, which was synthesised in vitro ${ }^{30,31}$, were mixed at a 3:1 ratio and injected $(1 \mathrm{nl})$ into fertilised eggs at the single-cell stage. The effect of the transgene on skin pattern formation was determined in F1 generation transgenic fish.

1. Kumar, N. M. \& Gilula, N. B. The gap junction communication channel. Cell 84, 381-388 (1996).

2. Simon, A. M. \& Goodenough, D. A. Diverse functions of vertebrate gap junctions. Trends Cell Biol. 8, 477-483 (1998).

3. Saez, J. C., Berthoud, V. M., Branes, M. C., Martinez, A. D. \& Beyer, E. C. Plasma membrane channels formed by connexins: their regulation and functions. Physiol. Rev. 83, 1359-1400 (2003)

4. Purnick, P. E., Benjamin, D. C., Verselis, V. K., Bargiello, T. A. \& Dowd, T. L. Structure of the amino terminus of a gap junction protein. Arch. Biochem. Biophys. 381, 181-190 (2000).

5. Oshima, A., Tani, K., Hiroaki, Y., Fujiyoshi, Y. \& Sosinsky, G. E. Threedimensional structure of a human connexin 26 gap junction channel reveals a plug in the vestibule. Proc. Natl. Acad. Sci. USA 104, 10034-10039 (2007).

6. Maeda, S. et al. Structure of the connexin 26 gap junction channel at $3.5 \AA$ resolution. Nature 458, 597-602 (2009).

7. Nakagawa, S., Maeda, S. \& Tsukihara, T. Structural and functional studies of gap junction channels. Curr. Opin. Struct. Biol. 20, 423-430 (2010).

8. Oh, S., Rubin, J. B., Bennett, M. V., Verselis, V. K. \& Bargiello, T. A. Molecular determinants of electrical rectification of single channel conductance in gap junctions formed by connexins 26 and 32. J. Gen. Physiol. 114, 339-364 (1999).

9. Musa, H. \& Veenstra, R. D. Voltage-dependent blockade of connexin40 gap junctions by spermine. Biophys. J. 84, 205-219 (2003).

10. Musa, H. et al. Amino terminal glutamate residues confer spermine sensitivity and affect voltage gating and channel conductance of rat connexin 40 gap junctions. $J$. Physiol. 557, 863-878 (2004).

11. Gemel, J., Lin, X., Veenstra, R. D. \& Beyer, E. C. N-terminal residues in Cx43 and $\mathrm{Cx} 40$ determine physiological properties of gap junction channels, but do not influence heteromeric assembly with each other or with Cx26. J. Cell Sci. 119, $2258-2268$ (2006)

12. Hibino, H. et al. Inwardly rectifying potassium channels: their structure, function, and physiological roles. Physiol. Rev. 90, 291-366 (2010).

13. Perrais, D., Veran, J. \& Mulle, C. Gating and permeation of kainate receptors: differences unveiled. Trends Pharmacol. Sci. 31, 516-522 (2010).

14. Beis, D. \& Stainier, D. Y. In vivo cell biology: following the zebrafish trend. Trends Cell Biol. 16, 105-112 (2006).

15. Parichy, D. M. et al. Mutational analysis of endothelin receptor b1 (rose) during neural crest and pigment pattern development in the zebrafish Danio rerio. Dev. Biol. 227, 294-306 (2000).

16. Lister, J. A., Robertson, C. P., Lepage, T., Johnson, S. L. \& Raible, D. W. nacre encodes a zebrafish microphthalmia-related protein that regulates neural-crestderived pigment cell fate. Development 126, 3757-3767 (1999).

17. Rawls, J. F. \& Johnson, S. L. Zebrafish kit mutation reveals primary and secondary regulation of melanocyte development during fin stripe regeneration. Development 127, 3715-3724 (2000).

18. Parichy, D. M., Ransom, D. G., Paw, B., Zon, L. I. \& Johnson, S. L. An orthologue of the kit-related gene fms is required for development of neural crest-derived xanthophores and a subpopulation of adult melanocytes in the zebrafish, Danio rerio. Development 127, 3031-3044 (2000).

19. Kondo, S. \& Miura, T. Reaction-diffusion model as a framework for understanding biological pattern formation. Science 329, 1616-1620 (2010).

20. Asai, R., Taguchi, E., Kume, Y., Saito, M. \& Kondo, S. Zebrafish leopard gene as a component of the putative reaction-diffusion system. Mech. Dev. 89, 87-92 (1999).

21. Yamaguchi, M., Yoshimoto, E. \& Kondo, S. Pattern regulation in the stripe of zebrafish suggests an underlying dynamic and autonomous mechanism. Proc. Natl. Acad. Sci. USA 104, 4790-4793 (2007).

22. Nakamasu, A., Takahashi, G., Kanbe, A. \& Kondo, S. Interactions between zebrafish pigment cells responsible for the generation of Turing patterns. Proc. Natl. Acad. Sci. USA 106, 8429-8434 (2009).

23. Kondo, S., Iwashita, M. \& Yamaguchi, M. How animals get their skin patterns: fish pigment pattern as a live Turing wave. Int. J. Dev. Biol. 53, 851-856 (2009).

24. Takahashi, G. \& Kondo, S. Melanophores in the stripes of adult zebrafish do not have the nature to gather, but disperse when they have the space to move. Pigment Cell \& Melanoma Res. 21, 677-686 (2008).
25. Iwashita, M. et al. Pigment pattern in jaguar/obelix zebrafish is caused by a Kir7.1 mutation: implications for the regulation of melanosome movement. PLoS Genet. 2, e197 (2006).

26. Inaba, M., Yamanaka, H. \& Kondo, S. Pigment pattern formation by contactdependent depolarization. Science, 335, 677 (2011).

27. Maderspacher, F. \& Nusslein-Volhard, C. Formation of the adult pigment pattern in zebrafish requires leopard and obelix dependent cell interactions. Development 130, 3447-3457 (2003).

28. Watanabe, M. et al. Spot pattern of leopard Danio is caused by mutation in the zebrafish connexin41.8 gene. EMBO Rep. 7, 893-897 (2006).

29. Watanabe, M. \& Kondo, S. Changing clothes easily: connexin41.8 regulates skin pattern variation. Pigment Cell \& Melanoma Res., 25, 326-330 (2012).

30. Kawakami, K. Transposon tools and methods in zebrafish. Dev. Dyn. 234, 244-254 (2005)

31. Kawakami, K., Shima, A. \& Kawakami, N. Identification of a functional transposase of the Tol2 element, an Ac-like element from the Japanese medaka fish, and its transposition in the zebrafish germ lineage. Proc. Natl. Acad. Sci. USA 97, 11403-11408 (2000).

32. Eastman, S. D., Chen, T. H., Falk, M. M., Mendelson, T. C. \& Iovine, M. K. Phylogenetic analysis of three complete gap junction gene families reveals lineagespecific duplications and highly supported gene classes. Genomics 87, 265-274 (2006).

33. Lin, H. J., Lien, Y. C. \& Hsu, C. H. A high-throughput colorimetric assay to characterize the enzyme kinetic and cellular activity of spermidine/spermine N1acetyltransferase 1. Anal. Biochem. 407, 226-232 (2010).

34. Gerhart, S. V., Jefferis, R. \& Iovine, M. K. Cx40.8, a Cx43-like protein, forms gap junction channels inefficiently and may require $\mathrm{Cx} 43$ for its association at the plasma membrane. FEBS Letters 583, 3419-3424 (2009).

35. Harris, A. L. Connexin channel permeability to cytoplasmic molecules. Prog. Biophys. Mol. Biol. 94, 120-143 (2007).

36. Kibschull, M., Gellhaus, A. \& Winterhager, E. Analogous and unique functions of connexins in mouse and human placental development. Placenta 29, 848-854 (2008).

37. Pfenniger, A., Wohlwend, A. \& Kwak, B. R. Mutations in connexin genes and disease. Eur. J. Clin. Invest. 41, 103-116 (2010).

38. Mathias, R. T., White, T. W. \& Gong, X. Lens gap junctions in growth, differentiation, and homeostasis. Physiol. Rev. 90, 179-206 (2010).

39. Scott, C. A. \& Kelsell, D. P. Key functions for gap junctions in skin and hearing. Biochem. J. 438, 245-254 (2011).

40. Scott, C. A., Tattersall, D., O’Toole, E. A. \& Kelsell, D. P. Connexins in epidermal homeostasis and skin disease. Biochim. Biophys. Acta doi:10.1016/ j.bbamem.2011.1009.1004 (2011).

41. Abrams, C. K. \& Scherer, S. S. Gap junctions in inherited human disorders of the central nervous system. Biochim. Biophys. Acta doi:10.1016/ j.bbamem.2011.1008.1015 (2011).

42. Schweda, F. et al. Substitution of connexin 40 with connexin 45 prevents hyperreninemia and attenuates hypertension. Kidney Int. 75, 482-489 (2009).

43. Westerfield, M. The zebrafish book: A guide for the laboratory use of zebrafish (Danio rerio). (University of Oregon Press, 1995).

\section{Acknowledgments}

We thank Prof. K. Kawakami at the National Institute of Genetics for providing the Tol2 plasmid and Mr. Hanzawa at Osaka University for technical assistance in generating transgenic fish. We also thank Profs. Y. Kurachi and K. Furutani at Graduate School of Medicine, Osaka University for helpful discussions on the electrophysiological analysis of gap junctions. This work was supported by a Grant-in-Aid for Young Scientists (B) to M.W. and a Grant-in-Aid for Scientific Research on Innovative Areas to S.K. from the Ministry of Education, Culture, Sports, Science and Technology of Japan.

\section{Author contributions}

M.W. and S.K. designed and M.W. and D.W. performed the research; M.W., D.W. and S.K. analysed data; M.W. wrote the paper.

\section{Additional information}

Supplementary information accompanies this paper at http://www.nature.com/ scientificreports

Competing financial interests: The authors declare no competing financial interests.

License: This work is licensed under a Creative Commons

Attribution-NonCommercial-NoDerivative Works 3.0 Unported License. To view a copy of this license, visit http://creativecommons.org/licenses/by-nc-nd/3.0/

How to cite this article: Watanabe, M., Watanabe, D. \& Kondo, S. Polyamine sensitivity of gap junctions is required for skin pattern formation in zebrafish. Sci. Rep. 2, 473; DOI:10.1038/srep00473 (2012) 